Pacific Journal of Mathematics

CONFORMAL CLUSTER SETS AND BOUNDARY CLUSTER 


\title{
CONFORMAL CLUSTER SETS AND BOUNDARY CLUSTER SETS COINCIDE
}

\author{
RAIMO NÄKKI
}

\begin{abstract}
The result stated in the title is proved. No restriction other than the obvious requirement that the cluster sets be taken at a nonisolated boundary point is imposed on the domain where the mapping is defined. The result is then generalized by allowing for certain exceptional sets on the boundary. More refined versions are established in the special case where the domain is the open unit disk. These include the statement that one-sided cluster sets coincide with one-sided radial cluster sets. Again, certain exceptional sets on the boundary are allowed for. Consequences are presented in which the existence of limits along sets on the boundary implies limits inside the domain. Finally, generalizations to the class of homeomorphisms satisfying the Carathéodory Prime End Theorem are indicated.
\end{abstract}

1. Introduction. In this paper we show that

$$
C(f, b)=C_{\partial D}(f, b)
$$

for a conformal mapping $f$ of a domain $D$ in the extended complex plane $\overline{\mathbb{C}}$. Here $C(f, b)$ denotes the cluster set of $f$ at a boundary point $b$ of $D$, while $C_{\partial D}(f, b)$ denotes the boundary cluster set of $f$ at $b$ (see $\S 2$ for definitions). We impose no restrictions on the domain $D$ other than the requirement that the boundary point $b$ be non-isolated, which is essential if $C_{\partial D}(f, b)$ is to be nonempty. Thus $D$ can be of finite or infinite connectivity. In $\S 3$ relation (1) is generalized to

$$
C(f, b)=C_{\partial D-E}(f, b)
$$

allowing for certain exceptional sets $E$ on $\partial D$. For instance, a condition guaranteeing (2) will be given in terms of logarithmic measure.

The much studied case where $D$ is the open unit disk $B$ will be considered next. Several extensions of the classical theorem of Iversen and Tsuji [4, p. 91] will be presented in the conformal case. For instance, we establish (2), with $D=B$, for any set $E$ on $\partial B$ satisfying one of the following six conditions:

$1^{\circ} . E$ is of capacity zero.

$2^{\circ}$. $E$ is of linear measure zero.

$3^{\circ} . E$ is an $O_{A D^{-}}$set. 
$4^{\circ}$. The logarithmic measure of $\partial B-E$ is infinite at each point of $E$ near $b$.

$5^{\circ}$. The radial density of $\partial B-E$ is positive at each point of $E$ near $b$.

$6^{\circ}$. The capacity density of $\partial B-E$ is positive at each point of $E$ near $b$.

As an example we exhibit a set $E$ on $\partial B$ with full measure, in fact with Hausdorff-dim $(\partial B-E)=0$, such that (2) holds for each point $b$ in $\partial B$ and for each conformal mapping $f$ of $B$.

In $\S 5$ relation (1) is further generalized by considering radial cluster sets instead of full cluster sets. We show that conformal cluster sets and radial boundary cluster sets coincide. More specifically,

$$
C(f, b)=C_{\mathrm{rad}, \partial B-E}(f, b)
$$

for any set $E$ on $\partial B$ satisfying one of the conditions $1^{\circ}-6^{\circ}$ above. One-sided versions of (3) will also be formulated. In $\S 6$ we observe, furthermore, that the induced radial extension $\dot{f}$ of $f$ satisfies

$$
C(f, b)=C\left(\dot{f} \mid\left(A_{f}-E\right), b\right) .
$$

Here $A_{f}$ denotes the set of boundary points of $B$ at which $f$ has a radial limit, while $E$ designates again an exceptional set on $\partial B$ satisfying one of the conditions $1^{\circ}-6^{\circ}$ above.

As consequences of relations (1)-(4) we obtain several generalizations of a classical theorem of Lindelöf $[12$, p. 75] in which the existence of limits along sets on the boundary implies total limits. These are presented in $\S 8$. Finally in $\S 9$ we formulate a topological version of our main theorem. Namely, we show, in the case where $D$ is the unit disk, that (1) holds for any homeomorphism satisfying the Carathéodory Prime End Theorem. The author would like to thank the referee for suggesting this generalization.

2. Cluster sets and boundary cluster sets. Let $f$ be a mapping defined in a domain $D$ in the extended complex plane $\overline{\mathbb{C}}$ and let $b$ be a boundary point of $D$. The cluster set of $f$ at $b$ is defined as

$$
C(f, b)=\bigcap_{U} \overline{f(U \cap D)},
$$

where $U$ ranges over all neighborhoods of $b$. The cluster set of $f$ on a nonempty set $E \subset \partial D$ is defined as

$$
C(f, E)=\bigcup_{b \in E} C(f, b) .
$$


The boundary cluster set of $f$ at $b$ is defined as

$$
C_{\partial D}(f, b)=\bigcap_{U} \overline{C(f, U \cap \partial D-\{b\})},
$$

where $U$ again ranges over all neighborhoods of $b$. The boundary cluster set of $f$ at $b$ along a set $F \subset \partial D$ is defined as

$$
C_{F}(f, b)=\bigcap_{U} \overline{C(f, U \cap F-\{b\})} .
$$

Obviously $C(f, b), C_{\partial D}(f, b)$ and $C_{F}(f, b)$ are closed sets,

$$
C_{F}(f, b) \subset C_{\partial D}(f, b) \subset C(f, b)
$$

and $C(f, b)$ is always nonempty. The boundary cluster set $C_{\partial D}(f, b)$ is nonempty if and only if $b$ is a non-isolated boundary point of $D$. It is evident that $C(f, b)$ may differ from $C_{\partial D}(f, b)$ even in the case where $D$ is the unit disk $B$ and $f$ is analytic in $B$. A standard example is given by

$$
f(z)=e^{(z+1) /(z-1)},
$$

which defines a bounded analytic function in the disk $B$ with $C(f, 1)=$ $\bar{B}$ and $C_{\partial B}(f, 1)=\partial B$. It is also easy to construct a homeomorphism $f$ of $B$ in such a fashion that $C_{\partial B}(f, b)$ is a proper subset of $C(f, b)$. For instance, if $g$ is a conformal mapping of $B$ onto the open triangle $G$ with vertices at 0,1 and $i$, chosen so that the induced boundary extension keeps the point 1 fixed, and if $h$ is the homeomorphism of $G$ which in vertical direction linearly stretches $G$ onto the square $H$ with vertices at $0,1,1+i$ and $i$, then $f=h \circ g$ is a homeomorphism of $B$ onto $H$ such that the boundary cluster set $C_{\partial B}(f, 1)$ consists of two points, 1 and $1+i$, while the cluster set $C(f, 1)$ quite clearly is the whole closed line segment connecting 1 to $1+i$. In this example, therefore, $C_{\partial B}(f, 1)$ is a proper subset of $C(f, 1)$. On the other hand, by a result due to Collingwood [3], [4, p. 82], there can exist no more than countably many points $b$ on $\partial B$ for which $C(f, b)$ and $C_{\partial B}(f, b)$ differ, even if $f$ is an arbitrary complex-valued function defined on $B$.

Our main result asserts that in the event the mapping $f$ is conformal, the sets $C(f, b)$ and $C_{\partial D}(f, b)$ coincide for all $b$, not merely in the case where $D$ is the unit disk $B$ [6], but for an arbitrary domain $D$ as wellthe trivial exceptions being, of course, the isolated points $b$ of $\partial D$ :

THEOREM 1. Let $b$ be a non-isolated boundary point of a domain D and let $f$ be a conformal mapping of $D$. Then

$$
C(f, b)=C_{\partial D}(f, b) \text {. }
$$


Proof. Suppose, contrary to the assertion, that there is a point $b^{\prime}$ in $C(f, b)-C_{\partial D}(f, b)$. After applying chordal isometries, we may assume that $b$ and $b^{\prime}$ are finite points. Since $C_{\partial D}(f, b)$ is closed,

$$
\operatorname{dist}\left[b^{\prime}, C_{\partial D}(f, b)\right]>0 .
$$

Let $B^{\prime}$ be an open disk centered at $b^{\prime}$ such that

$$
\overline{B^{\prime}} \cap C_{\partial D}(f, b)=\varnothing,
$$

let $V$ be a neighborhood of $C_{\partial D}(f, b)$ satisfying

$$
\overline{B^{\prime}} \cap \bar{V}=\varnothing,
$$

let $U$ be a neighborhood of $b$ for which

$$
C(f, U \cap \partial D-\{b\}) \subset V
$$

and let $\left\langle b_{k}\right\rangle$ be a sequence of points in $D$ converging to $b$ such that

$$
\lim f\left(b_{k}\right)=b^{\prime} \text {. }
$$

We may assume that $f\left(b_{k}\right) \in B^{\prime}$ for each $k$.

Fix a point $c^{\prime}$ in $B^{\prime} \cap f(D)$. For each $k$ choose a closed line segment $\alpha_{k}^{\prime}$ in $B^{\prime}$ with endpoints $c^{\prime}$ and $f\left(b_{k}\right)$. Designate by $\beta_{k}^{\prime}$ the component of $\alpha_{k}^{\prime} \cap f(D)$ which contains $f\left(b_{k}\right)$ and set $\beta_{k}=f^{-1}\left(\beta_{k}^{\prime}\right)$. If $\beta_{k}^{\prime}=\alpha_{k}^{\prime}$, then $\beta_{k}$ is a closed Jordan arc in $D$ joining $b_{k}$ to $c=f^{-1}\left(c^{\prime}\right)$. If $\beta_{k}^{\prime} \neq \alpha_{k}^{\prime}$, then $\beta_{k}^{\prime}$ is a line segment with an endpoint on the boundary of $f(D)$ and, therefore, $\beta_{k}$ is a Jordan arc in $D$ with an accumulation point in $\partial D$. In view of (5) and (6), any such accumulation point must either lie in $\partial D-U$ or coincide with $b$. In each case, $\beta_{k}$ is a Jordan arc in $D$ containing $b_{k}$. Moreover, either $\beta_{k}$ contains the point $c$ or $\beta_{k}$ accumulates at $\{b\} \cup(\partial D-U)$.

Let $J$ denote the set of all numbers $r$ on $(0,1)$ such that $S(b, r)$, the circle of radius $r$ centered at $b$, intersects $\partial D$. We claim that

$$
\int_{J} \frac{d r}{r}=\infty
$$

If (7) were not true, then

$$
\int_{(0,1)-J} \frac{d r}{r}=\infty
$$

and, consequently, the family of circles centered at $b$ and lying in $D$ would have infinite modulus (that is, zero extremal length). Thus $f$ would have a limit at $b$. (See, for example, Grötzsch [8] and Jurchescu 
[9].) But this means that $C(f, b)$ would be degenerate and would thereby coincide with $C_{\partial D}(f, b)$. We conclude that (7) must hold.

Next consider the family $\Gamma_{k}$ of open arcs joining $\beta_{k}$ to $U \cap \partial D-\{b\}$ in $D$. If $b$ is an accumulation point of $\beta_{k}$, then the minorization principle of the modulus in combination with (7) evidently yields $\bmod \left(\Gamma_{k}\right)=\infty$. If $\beta_{k}$ contains $c$ for arbitrarily large $k$, condition (7) enables us to fix an index $k_{0}$ such that $\Gamma_{k_{0}}$ has arbitrarily large modulus, say

$$
\bmod \left(\Gamma_{k_{0}}\right)>M,
$$

where $M$ designates the modulus of the family $\Gamma^{\prime}$ of arcs joining $\overline{B^{\prime}}$ to $\bar{V}$ in $\mathbb{C}$. Observe that $M<\infty$ by virtue of (5). If $\beta_{k}$ has an accumulation point in $\partial D-U$, then again, as above, we can fix an index $k_{0}$ such that (8) holds. As pointed out earlier, one of these three possibilities must be true of $\beta_{k}$. We conclude that (8) is satisfied for some $k_{0}$.

On the other hand, for each $k$ the family $f\left(\Gamma_{k}\right)$ is contained in $\Gamma^{\prime}$. We infer, in particular, that

$$
\bmod \left[f\left(\Gamma_{k_{0}}\right)\right] \leq M .
$$

But since $\bmod \left(\Gamma_{k_{0}}\right)=\bmod \left[f\left(\Gamma_{k_{0}}\right)\right]$ by virtue of the conformal invariance of the modulus, inequalities (8) and (9) yield the desired contradiction. The proof is complete.

3. Relation $C(f, b)=C_{\partial D-E}(f, b)$. Theorem 1 is subject to various generalizations. One such generalization is obtained by permitting exceptional sets $E$ on $\partial D$ and looking for conditions under which

$$
C(f, b)=C_{\partial D-E}(f, b) .
$$

An examination of the proof of Theorem 1 suggests the introduction of the following concept to describe the denseness of a set $A$ relative to a domain $D$.

Let $A$ be a nonempty set on the boundary of a domain $D$ and let $z \in \partial D, z \neq \infty$. We will write

$$
\bmod _{D}(A, z)=\infty
$$

if, for each $r>0$,

$$
\liminf _{t \rightarrow 0} \bmod [\Delta(A, F: D)]=\infty,
$$

where $\Delta(A, F: D)$ designates the family of all open arcs in $D$ having one endpoint in $A$ and the other in $F$ and where the infimum is extended over all continua $F$ in $D$ meeting the circles $S(z, r)$ and $S(z, t)$. 
If $z=\infty$, the above concept is defined via an inversion. Condition (10) is equivalent to the requirement that, for each $r>0$,

$$
\liminf _{t \rightarrow 0} \bmod [\Delta(A \cap B(z, r), F: D)]=\infty .
$$

Here $B(z, r)$ denotes the open disk of radius $r$ centered at $z$ and $F$ is as before.

In the special case where $A$ is compact and $D$ is the whole space, condition (10) was first used by Martio [10] to describe the thickness of a set $A$ at a point $z$.

THEOREM 2. Let $b$ be a non-isolated boundary point of a domain $D$ and let $E$ be a set on $\partial D$ such that $\bmod _{D}(\partial D-E, z)=\infty$ for each point $z$ in $E \cup\{b\}$ near $b$. Next let $f$ be a conformal mapping of $D$. Then

$$
C(f, b)=C_{\partial D-E}(f, b) .
$$

Proof. The proof of Theorem 1 requires only cosmetic changes. Let $U_{0}$ be a neighborhood of $b$ such that $\bmod _{D}(\partial D-E, z)=\infty$ for each $z$ in $U_{0} \cap(E \cup\{b\})$. Replace $\partial D$ by $\partial D-E$ in the proof of Theorem 1 , choose the neighborhood $U$ in (6) to be a subset of $U_{0}$ and let otherwise $b^{\prime}, b_{k}, c, c^{\prime}, \alpha_{k}^{\prime}, \beta_{k}^{\prime}$ and $\beta_{k}$ be as in the proof of Theorem 1. In the present situation $\beta_{k}$ is a Jordan arc in $D$ containing $b_{k}$, and, either $\beta_{k}$ contains $c$ or $\beta_{k}$ accumulates at $\{b\} \cup(U \cap E) \cup(\partial D-U)$. We let $\Gamma_{k}$ denote the family of open arcs joining $\beta_{k}$ to $U \cap(\partial D-E)-\{b\}$ in $D$ and use the $\bmod _{D}$-hypothesis, in conjunction with (11), to conclude that the modulus estimate (8) holds. Since (9) remains valid, the assertion follows.

A more concrete condition guaranteeing the conclusion of Theorem 2 will be provided by our next result:

THEOREM 3. Let $f$ be a conformal mapping of a domain $D$ and let $b \neq \infty$ be a non-isolated boundary point of $D$. Next let $E$ be a set on $\partial D$ such that, for each $z$ in $E \cup\{b\}$ near $b$, the set $J_{z}$ of all numbers $r$ on $(0,1)$ for which $S(z, r)$ meets $\partial D$ and omits $E$ is measurable and satisfies

$$
\int_{J_{z}} \frac{d r}{r}=\infty
$$

Then

$$
C(f, b)=C_{\partial D-E}(f, b)
$$


Proof. Fix $z$ in $E \cup\{b\}$ satisfying (12). We show that

$$
\bmod _{D}(\partial D-E, z)=\infty \text {. }
$$

Fix $r$ in $(0,1)$ and $M>0$. By virtue of (12), we may choose $t$ on $(0, r)$ so that

$$
\int_{J_{z} \cap(t, r)} \frac{d u}{u} \geq 2 \pi M .
$$

Let $F$ be a continuum in $D$ which meets both $S(z, r)$ and $S(z, t)$, let $\Gamma$ be the family of open arcs in $D$ that join $\partial D-E$ to $F$, and let $\rho$ be an admissible density for $\Gamma$. For $u$ in $J_{z}$, the circle $S_{u}=S(z, u)$ contains an arc $\gamma_{u}$ which belongs to $\Gamma$. Thus, by Schwarz's inequality,

$$
1 \leq\left(\int_{\gamma_{u}} \rho|d z|\right)^{2} \leq 2 \pi u \int_{S_{u}} \rho^{2}|d z|,
$$

and hence

$$
\int_{S_{u}} \rho^{2}|d z| \geq \frac{1}{2 \pi u}
$$

This inequality is true for all $u$ in $J_{z} \cap(t, r)$. Integrating over $J_{z} \cap(t, r)$ yields, in light of (13),

$$
\int_{\mathbb{C}} \rho^{2} d x d y \geq \int_{J_{z} \cap(t, r)}\left(\int \rho^{2}|d z|\right) d u \geq M .
$$

Since $\rho$ was an arbitrary density for $\Gamma,(14) \operatorname{gives} \bmod (\Gamma) \geq M$. We conclude that $\bmod _{D}(\partial D-E, z)=\infty$.

Since $\bmod _{D}(\partial D-E, z)=\infty$ for each point $z$ in $E \cup\{b\}$ near $b$, the assertion follows from Theorem 2 .

4. One-sided cluster sets in the case $D=B$. In this section we present a refinement of Theorem 1 in the special case where $D$ is the open unit disk $B$.

Let $f$ be a mapping defined in $B$ and let $b$ be a point in $\partial B$. The left-hand cluster set of $f$ at $b$ is defined as

$$
\begin{aligned}
C_{L}(f, b)=\left\{b^{\prime} \in \overline{\mathbb{C}}: f\left(b_{k}\right) \rightarrow b^{\prime}\right. & \text { for some sequence }\left\langle b_{k}\right\rangle \\
& \text { in } \left.B \text { with } b_{k} \rightarrow b, \arg b_{k} \geq \arg b\right\} .
\end{aligned}
$$

The right-hand cluster set $C_{R}(f, b)$ of $f$ at $b$ is defined analogously. The left-hand boundary cluster set of $f$ at $b$ is defined as

$$
C_{L, \partial B}(f, b)=\bigcap_{U} \overline{C\left(f, U_{-} \cap \partial B-\{b\}\right)},
$$


where $U$ ranges over all neighborhoods of $b$ and where $U_{-}=\{z \in$ $U: \arg z \geq \arg b\}$. The right-hand boundary cluster set $C_{R, \partial B}(f, b)$ is defined analogously. The left-hand boundary cluster set of $f$ at $b$ along a set $F \subset \partial B$ is defined as

$$
C_{L, F}(f, b)=\bigcap_{U} \overline{C\left(f, U_{-} \cap F-\{b\}\right)} .
$$

The corresponding right-hand boundary cluster set $C_{R, F}(f, b)$ along $F$ is defined in a similar fashion.

Obviously,

$$
C_{L, F}(f, b) \subset C_{L, \partial B}(f, b) \subset C_{L}(f, b) .
$$

REMARK 1. In the theory of prime ends a different terminology is frequently used when referring to some of the cluster sets defined above. (For pertinent notions of the prime end theory, consult [2], [4, Chapter 9] and [13].) To wit, let $f$ be a conformal mapping of $B$, let $b$ be a point of $\partial B$ and let $\mathscr{P}$ be the prime end of $f(B)$ corresponding to $b$ under $f$ in the sense of the Carathéodory Prime End Theorem. Denote by $I(\mathscr{P})$ the impression of $\mathscr{P}$. Then

$$
I(\mathscr{P})=C(f, b) .
$$

The left-hand cluster set $C_{L}(f, b)$ is sometimes called the left wing of $I(\mathscr{P})$, while $C_{R}(f, b)$ is the right wing of $I(\mathscr{P})$. Next let $\pi(\mathscr{P})$ denote the set of principal points of $I(\mathscr{P})$, that is, $\pi(\mathscr{P})$ is the set of limit points of convergent chains in $\mathscr{P}$. Then $\pi(\mathscr{P})$ is the intersection of the left and right wings of $I(\mathscr{P})$,

$$
\pi(\mathscr{P})=C_{L}(f, b) \cap C_{R}(f, b) .
$$

One may also express $\pi(\mathscr{P})$ as

$$
\pi(\mathscr{P})=C_{\text {rad }}(f, b),
$$

where $C_{\text {rad }}(f, b)$ designates the cluster set of $f$ at $b$ taken along the radius of $B$ terminating at $b$. The points in $I(\mathscr{P})-\pi(\mathscr{P})$, if they exist, are called subsidiary points of $I(\mathscr{P})$.

Collingwood has shown, for an arbitrary complex-valued function $f$ defined on $B$, that

$$
C_{L}(f, b)=C_{L, \partial B}(f, b),
$$

except perhaps for a countable set of points $b$ on $\partial B$. Even more surprisingly,

$$
C_{L}(f, b)=C_{R}(f, b)
$$


for such an $f$, except perhaps for a countable set of points $b$ on $\partial B$, $[3],[4$, p. 82]. The exceptional points $b$ in the statement (18) cannot, in general, be eliminated, even if the condition of regularity or conformality on $f$ were to be imposed. Examples manifesting this are not difficult to construct. (For instance, map $B$ conformally onto a suitable comb-domain having a countable number of prime ends of the second kind. More elaborate examples may be found in [5].) The relation (17), on the other hand, although sharp even for bounded analytic functions [6], renders itself to considerable improvement in the conformal case: no exceptional points $b$ can exist. We prove, in fact, the following extension of the Iversen-Tsuji Theorem [4, p. 91]:

THeOREM 4. Let $b$ be a boundary point of the unit disk $B$ and let $E$ be a set on $\partial B$ such that $\bmod _{B}(\partial B-E, z)=\infty$ for each point $z$ in $E$ near $b$. Next let $f$ be a conformal mapping of $B$. Then

$$
\begin{aligned}
& C_{L}(f, b)=C_{L, \partial B-E}(f, b), \\
& C_{R}(f, b)=C_{R, \partial B-E}(f, b) .
\end{aligned}
$$

Proof. We show that $C_{L}(f, b)=C_{L, \partial B-E}(f, b)$. By virtue of (15), it is sufficient to verify the inclusion $C_{L}(f, b) \subset C_{L, \partial B-E}(f, b)$. Suppose, contrary to the assertion, that there is a point $b^{\prime}$ in $C_{L}(f, b)-$ $C_{L, \partial B-E}(f, b)$. We may assume that $b=i$ and $b^{\prime} \neq \infty$. Since $C_{L, \partial B-E}(f, b)$ is closed,

$$
\operatorname{dist}\left(b^{\prime}, C_{L, \partial B-E}(f, b)\right)>0 .
$$

Let $B^{\prime}$ be an open disk centered at $b^{\prime}$ such that

$$
\bar{B}^{\prime} \cap C_{L, \partial B-E}(f, b)=\varnothing,
$$

let $V$ be a neighborhood of $C_{L, \partial B-E}(f, b)$ satisfying

$$
\bar{B}^{\prime} \cap \bar{V}=\varnothing \text {, }
$$

and let $U$ be a neighborhood of $b$ for which

$$
C\left(f, U_{-} \cap(\partial B-E)-\{b\}\right) \subset V,
$$

where $U_{-}=\{z \in U: \arg z \geq \arg b\}$. Next let $\left\langle b_{k}\right\rangle$ be a sequence of points in $B_{-}=\{z \in B: \operatorname{Re} z \leq 0\}$ converging to $b$ so that

$$
\lim b_{k}^{\prime}=b^{\prime} \text {, }
$$

where $b_{k}^{\prime}=f\left(b_{k}\right)$. We may assume that $b_{k}^{\prime} \in B^{\prime}$ for each $k$. Finally, let $\mathscr{P}$ be the prime end of $D=f(B)$ corresponding to $b$ under $f$ in the 
sense of the Carathéodory Prime End Theorem. We divide the proof into two parts according to whether or not $b^{\prime}$ is a principal point of the impression $I(\mathscr{P})$.

Assume first that $b^{\prime}$ is a principal point of $I(\mathscr{P})$. Thus $b^{\prime}$ is the limit of a chain of cross-cuts $q_{k}$ of $D$ belonging to $\mathscr{P}$ with

$$
\operatorname{dia}\left(q_{k}\right) \rightarrow 0 \text {. }
$$

It is a classical result, perhaps due to Tsuji, that each $q_{k}$ in (21) can be chosen to be an image under $f$ of a circular arc $B \cap S\left(b, r_{k}\right)$, where $r_{k} \rightarrow 0$. For each $k$, let

$$
\begin{aligned}
D_{k} & =f\left[B \cap B\left(b, r_{k}\right)\right], \\
D_{k}^{-} & =f\left[B-\cap B\left(b, r_{k}\right)\right] .
\end{aligned}
$$

Passing to subsequences and relabeling, we may assume that $q_{k}$ lies in $B^{\prime}$ and that $b_{k}^{\prime}$ lies in $D_{k}^{-}$for each $k$. Join $b_{k}^{\prime}$ to $q_{k}$ by an arc $\alpha_{k}$ which, except for the terminal point, lies in $D_{k}^{-}$. We can extend $\alpha_{k}$ into an end-cut $A_{k}$ of $D$ by adding to $\alpha_{k}$ a portion of $q_{k}$ which lies on the arc $f\left[B_{-} \cap S\left(b, r_{k}\right)\right]$. The pre-image $f^{-1}\left(A_{k}\right)$ is an end-cut of $B$ from $b_{k}$ and lies, except for the terminal point, $a_{k}$, in $B_{-} \cap \bar{B}\left(b, r_{k}\right)$. Clearly $a_{k} \rightarrow b$ and $a_{k} \neq b$ for each $k$. Since, by Lindelöf's theorem, an endpoint of $q_{k}$ is the radial limit of $f$ at $a_{k}$ and since $q_{k}$ lies in $B^{\prime}$, it follows from (19) that $a_{k}$ must lie in $E$ for each $k$.

Now consider the family $\Gamma_{k}$ of open arcs in $B$ with endpoints in $f^{-1}\left(A_{k}\right)$ and $U_{-} \cap(\partial B-E)-\{b\}$. Since $a_{k}$, the terminal point of $f^{-1}\left(A_{k}\right)$, lies in $E$ and since $a_{k} \neq b$, our hypotheses imply that

$$
\bmod \left(\Gamma_{k}\right)=\infty
$$

for each $k$. On the other hand, the arcs in the image family $\Gamma_{k}^{\prime}$ join $A_{k}$ to $C\left(f, U_{-} \cap(\partial B-E)-\{b\}\right)$ in $D$. The arc $A_{k}$ lies in $D_{k}^{-} \cup q_{k}$, but not necessarily in $\bar{B}^{\prime}$. Nevertheless, since $q_{k}$ lies in $B^{\prime}$ it follows from (19) and (20) that no arc in $\Gamma_{k}^{\prime}$ can avoid $q_{k}$. We combine (21) with the minorization principle for the extremal length and infer that

$$
\bmod \left(\Gamma_{k}^{\prime}\right) \rightarrow 0
$$

as $k \rightarrow \infty$. But conditions (22) and (23) contradict the conformal invariance of the extremal length.

It remains to derive a contradiction also in the case where $b^{\prime}$ is a subsidiary point of $I(\mathscr{P})$. Choose again a decreasing sequence of positive numbers $r_{k} \rightarrow 0$ such that the circular arcs $B \cap S\left(b, r_{k}\right)$ are mapped by $f$ onto disjoint cross-cuts $c_{k}$ of $D$ with the property that

$$
\operatorname{dia}\left(c_{k}\right) \rightarrow 0 \text {. }
$$


Obviously the cross-cuts $c_{k}$ form a chain in $\mathscr{P}$. However, they do not converge to $b^{\prime}$, because $b^{\prime}$ was a subsidiary point of $I(\mathscr{P})$. Let $D_{k}, D_{k}^{-}$and $B_{-}$be as earlier in the proof. Again we may assume that $b_{k}^{\prime}$ lies in $D_{k}^{-}$for each $k$. Join $b_{k}^{\prime}$ to $b^{\prime}$ by a line segment and denote by $b_{k}^{\prime \prime}$ the first point at which the segment, starting from $b_{k}^{\prime}$, meets $\partial D_{k}^{-}$. Obviously $b_{k}^{\prime \prime} \rightarrow b^{\prime}$. The point $b_{k}^{\prime \prime}$ cannot lie in $c_{k}$ for large $k$, because otherwise, in view of (24), $b^{\prime}$ would be a principal point of $I(\mathscr{P})$. Neither can $b_{k}^{\prime \prime}$, for large $k$, lie on the image under $f$ of the radius of $B$ terminating at $b$, because otherwise, in view of (16), $b^{\prime}$ would again be a principal point of $I(\mathscr{P})$. Thus $b_{k}^{\prime \prime}$ must lie on $\partial D$ for all sufficiently large $k$. We may assume this to be the case for all $k$. It is clear, furthermore, that $b_{k}^{\prime \prime} \neq b^{\prime}$ for each $k$, because otherwise $b^{\prime}$ would be accessible relative to the prime end $\mathscr{P}$ and again $b^{\prime}$ would be a principal point of $I(\mathscr{P})$. Denote by $L_{k}$ the open line segment with endpoints $b_{k}^{\prime}$ and $b_{k}^{\prime \prime}$.

By a classical theorem of Koebe, $f^{-1}$ has a limit $a_{k}$ at $b_{k}^{\prime \prime}$ along the line segment $L_{k}$. Since $f^{-1}\left(D_{k}\right) \rightarrow b$, it follows that $a_{k} \rightarrow b$. By Lindelöf's theorem, $b_{k}^{\prime \prime}$ is the radial limit of $f$ at $a_{k}$. Since $b_{k}^{\prime \prime} \rightarrow b^{\prime}$ and since the set $\pi(\mathscr{P})=C_{\text {rad }}(f, b)$ of principal points of $I(\mathscr{P})$ is closed and lies, therefore, at a positive distance from $b^{\prime}$, it follows that $a_{k} \neq b$ for all $k$ sufficiently large. We may assume that $a_{k} \neq b$ for all $k$. Since $b_{k}^{\prime \prime}$, the radial limit of $f$ at $a_{k}$, lies in $B^{\prime}$, it follows from (19) and (20) that $a_{k}$ must be located in $E$.

Now consider the family $\Delta_{k}$ of open arcs in $B$ with endpoints in $f^{-1}\left(L_{k}\right)$ and $U_{-} \cap(\partial B-E)-\{b\}$. Since $a_{k}$, the terminal point of $f^{-1}\left(L_{k}\right)$, lies in $E$ and since $a_{k} \neq b$, our hypotheses imply that

$$
\bmod \left(\Delta_{k}\right)=\infty
$$

for each $k$. Since $L_{k}$ is situated in $B^{\prime}$ and since $\operatorname{dia}\left(L_{k}\right) \rightarrow 0$, it follows from (19), (20) and the minorization principle for the extremal length that

$$
\bmod \left(\Delta_{k}^{\prime}\right) \rightarrow 0
$$

as $k \rightarrow \infty$, where $\Delta_{k}^{\prime}=f\left(\Delta_{k}\right)$. Again, (25) and (26) contradict the conformal invariance of the extremal length. The proof is complete.

5. Radial cluster sets and radial boundary cluster sets. We next further generalize Theorem 1 in the case $D=B$ by restricting the approach of $f$ to the boundary.

Let $f$ be a mapping of $B$, let $b \in \partial B$ and let

$$
\rho_{b}=\{t b: 0 \leq t<1\}
$$


be the radius in $B$ terminating at $b$. The radial cluster set of $f$ at $b$ is defined as

$$
C_{\text {rad }}(f, b)=C\left(f \mid \rho_{b}, b\right) .
$$

The radial cluster set of $f$ on a nonempty set $E \subset \partial B$ is defined as

$$
C_{\mathrm{rad}}(f, E)=\bigcup_{b \in E} C_{\mathrm{rad}}(f, b) .
$$

The radial boundary cluster set of $f$ at $b$ is defined as

$$
C_{\mathrm{rad}, \partial B}(f, b)=\bigcap_{U} \overline{C_{\mathrm{rad}}(f, U \cap \partial B-\{b\})},
$$

where $U$ ranges over all neighborhoods of $b$. The left-hand radial boundary cluster set of $f$ at $b$ is defined as

$$
C_{L, \mathrm{rad}, \partial B}(f, b)=\bigcap_{U} \overline{C_{\mathrm{rad}}\left(f, U_{-} \cap \partial B-\{b\}\right)},
$$

where $U$ is as above and where again $U_{-}=\{z \in U: \arg z \geq \arg b\}$. The right-hand radial boundary cluster set $C_{R, \mathrm{rad}, \partial B}(f, b)$ is defined analogously. The left-hand radial boundary cluster set of $f$ at $b$ along $a$ set $F \subset \partial B$ is defined as

$$
C_{L, \mathrm{rad}, F}(f, b)=\bigcap_{U} \overline{C_{\mathrm{rad}}\left(f, U_{-} \cap F-\{b\}\right)} .
$$

The corresponding right-hand radial boundary cluster set $C_{R, \mathrm{rad}, F}(f, b)$ along $F$ is defined in a similar fashion.

Obviously

$$
C_{L, \mathrm{rad}, F}(f, b) \subset C_{L, \mathrm{rad}, \partial B}(f, b) \subset C_{L, \partial B}(f, b) \subset C_{L}(f, b) .
$$

THEOREM 5. Let $b$ be a boundary point of the unit disk $B$ and let $E$ be a set on $\partial B$ such that $\bmod _{B}(\partial B-E, z)=\infty$ for each point $z$ in $E$ near $b$. Next let $f$ be a conformal mapping of $B$. Then

$$
\begin{aligned}
& C_{L}(f, b)=C_{L, \mathrm{rad}, \partial B-E}(f, b), \\
& C_{R}(f, b)=C_{R, \mathrm{rad}, \partial B-E}(f, b),
\end{aligned}
$$

and consequently,

$$
C(f, b)=C_{\mathrm{rad}, \partial B-E}(f, b) .
$$

Proof. We show that $C_{L}(f, b)=C_{L, \mathrm{rad}, \partial B-E}(f, b)$. Replace $C_{L, \partial B-E}(f, b)$ and $C\left(f, U_{-} \cap(\partial B-E)-\{b\}\right)$, respectively, by $C_{L, \mathrm{rad}, \partial B-E}(f, b)$ and $C_{\mathrm{rad}}\left(f, U_{-} \cap(\partial B-E)-\{b\}\right)$ in the proof of 
Theorem 4. Then proceed word by word and obtain the modulus estimate (22). Consider an arc $\gamma$ in $\Gamma_{k}$. Either $f(\gamma)$ is non-rectifiable or $f$ has a limit along $\gamma$ at the terminal point of $\gamma$. In the latter case, by virtue of Lindelöf's theorem, this limit is also the radial limit of $f$ and belongs, therefore, to $C_{\mathrm{rad}}\left(f, U_{-} \cap(\partial B-E)-\{b\}\right)$. Since the non-rectifiable paths do not effect the modulus, we conclude, as in the proof of Theorem 4, that (23) holds. This establishes the theorem in the case where $b^{\prime}$ is a principal point of $I(\mathscr{P})$.

In the remaining case $b^{\prime}$ is a subsidiary point of $I(\mathscr{P})$. The proof of Theorem 4 applies verbatim up to the modulus condition (25), while, in order to deduce the contradicting inequality (26), the observation must again be made that the arcs in $\Delta_{k}^{\prime}$ which do not have endpoints in $C_{\text {rad }}\left(f, U_{-} \cap(\partial B-E)-\{b\}\right)$ are non-rectifiable and, consequently, they do not influence the modulus of $\Delta_{k}^{\prime}$.

6. Radial extension and cluster sets. Let $f$ be a conformal mapping of $B$ and let $A_{f}$ be the set of points on $\partial B$ where $f$ has a radial limit, that is, where $C_{\text {rad }}(f, b)$ degenerates to a single point. We let $\dot{f}$ denote the radial extension of $f$ to $B \cup A_{f}$. Thus

$$
\dot{f}(x)= \begin{cases}f(x) & \text { if } x \in B, \\ \lim _{t \rightarrow 1} f(t x) & \text { if } x \in A_{f} .\end{cases}
$$

By a classical theorem of Beurling, $\partial B-A_{f}$ is of capacity zero.

Given a point $b$ on $\partial B$, the left-hand cluster set $C_{L}\left(\dot{f} \mid A_{f}, b\right)$ of the boundary mapping $\dot{f} \mid A_{f}$ at $b$ and the corresponding right-hand cluster set $C_{R}\left(\dot{f} \mid A_{f}, b\right)$ are defined in an obvious manner, as are the cluster sets $C_{L}(\dot{f} \mid F, b)$ and $C_{R}(\dot{f} \mid F, b)$ taken along a subset $F$ of $A_{f}$.

THeOREM 6. Let $b$ be a boundary point of the unit disk $B$ and let $E$ be a set on $\partial B$ such that $\bmod _{B}(\partial B-E, z)=\infty$ for each point $z$ in $E$ near $b$. Next let $f$ be a conformal mapping of $B$ and let $\dot{f}$ be the radial extension of $f$ to $B \cup A_{f}$. Then

$$
\begin{aligned}
& C_{L}(f, b)=C_{L}\left(\dot{f} \mid\left(A_{f}-E\right), b\right), \\
& C_{R}(f, b)=C_{R}\left(\dot{f} \mid\left(A_{f}-E\right), b\right),
\end{aligned}
$$

and consequently,

$$
C(f, b)=C\left(\dot{f} \mid\left(A_{f}-E\right), b\right)
$$

Proof. Every arc in $B$ with an endpoint in $\partial B-A_{f}$ is mapped by $f$ onto a non-rectifiable arc. The modulus of the family of all such 
arcs is, therefore, equal to zero. It follows that $\bmod _{B}\left(A_{f}-E, z\right)=\infty$ for each $z$ in $E$ near $b$, because by hypothesis, $\bmod _{B}(\partial B-E, z)=\infty$ for each such $z$. If we now replace $C_{L, \mathrm{rad}, \partial B-E}(f, b)$ and $C_{\text {rad }}\left(f, U_{-} \cap(\partial B-E)-\{b\}\right)$ respectively by $C\left(\dot{f} \mid\left(A_{f}-E\right), b\right)$ and $C\left(\dot{f}, U_{-} \cap\left(A_{f}-E\right)-\{b\}\right)$ in the proof of Theorem 5 , the proof applies verbatim.

7. Special cases. In Theorems 4-6, the exceptional set $E$ was assumed to satisfy $\bmod _{B}(\partial B-E, z)=\infty$ for all $z$ in $E$ near $b$. What kind of sets $E$ satisfy this condition? Several answers will be given in this section. We recall a number of pertinent notions.

Given a set $A$ and a point $z$ in $\mathbb{C}$, let $J_{z}$ denote the set of all numbers $r$ on $(0,1)$ for which the circle $S(z, r)$ intersects $A$. Assume that $J_{z}$ is measurable. If

$$
\int_{J_{z}} \frac{d r}{r}=\infty
$$

we write

$$
\log -\operatorname{meas}(A, z)=\infty \text {. }
$$

The upper radial density of $A$ at $z$ is defined as

$$
\operatorname{rad} \overline{\operatorname{dens}}(A, z)=\limsup _{r \rightarrow 0} \frac{l\left[J_{z} \cap(0, r)\right]}{r},
$$

where $l$ stands for linear measure. The upper capacity density of $A$ at $z$ is defined as

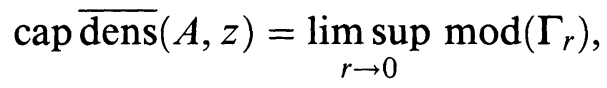

where $\Gamma_{r}=\Delta(A \cap \bar{B}(z, r), S(z, 2 r): \mathbb{C})$.

We also recall a concept due to Ahlfors and Beurling [1]. A set $A$ in $\overline{\mathbb{C}}$ is said to be an $O_{A D}$-set (or an NED-set) if

$$
\bmod \left[\Delta\left(F_{1}, F_{2}: \mathbb{C}\right)\right]=\bmod \left[\Delta\left(F_{1}, F_{2} ; \mathbb{C}-A\right)\right]
$$

for each pair of continua $F_{1}$ and $F_{2}$ in the complement of $A$. For a compact $A$, this is precisely the condition required to guarantee that every conformal mapping of $\overline{\mathbb{C}}-A$ admits an extension to a conformal mapping of $\overline{\mathbb{C}},[1]$.

THEOREM 7. Let $b$ be a boundary point of the unit disk $B$ and let $E$ be a set on $\partial B$ satisfying one of the following six conditions:

$1^{\circ}$. E is of capacity zero.

$2^{\circ}$. $E$ is of linear measure zero. 
$3^{\circ}$. E is an $O_{A D^{-} \text {set. }}$

$4^{\circ} . \log -\operatorname{meas}(\partial B-E, z)=\infty$ for each $z$ in $E$ near $b$.

$5^{\circ} \cdot \operatorname{rad} \overline{\operatorname{dens}}(\partial B-E, z)>0$ for each $z$ in $E$ near $b$.

$6^{\circ}$. cap $\overline{\operatorname{dens}}(\partial B-E, z)>0$ for each $z$ in $E$ near $b$.

Next let $f$ be a conformal mapping of $B$ and let $\dot{f}$ be the radial extension of $f$ to $B \cup A_{f}$. Then

$$
\begin{aligned}
& C_{L}(f, b)=C_{L, \partial B-E}(f, b)=C_{L, \mathrm{rad}, \partial B-E}(f, b)=C_{L}\left(\dot{f} \mid\left(A_{f}-E\right), b\right), \\
& C_{R}(f, b)=C_{R, \partial B-E}(f, b)=C_{R, \mathrm{rad}, \partial B-E}(f, b)=C_{R}\left(\dot{f} \mid\left(A_{f}-E\right), b\right),
\end{aligned}
$$

and consequently,

$$
C(f, b)=C_{\partial B-E}(f, b)=C_{\mathrm{rad}, \partial B-E}(f, b)=C\left(\dot{f} \mid\left(A_{f}-E\right), b\right) .
$$

Proof. In view of Theorems 4-6, it is sufficient to verify that each of the conditions $1^{\circ}-6^{\circ}$ implies $\bmod _{B}(\partial B-E, z)=\infty$ for every $z$ in $E$ near $b$.

It is classical that $1^{\circ}$ implies $2^{\circ}$, whereas it is obvious that $2^{\circ}$ implies $4^{\circ}$. We next show that $5^{\circ}$ implies $4^{\circ}$. For this, let $\operatorname{rad} \overline{\operatorname{dens}}(\partial B-E, z)=$ $\delta>0$ and let $J_{z}$ denote the set of all $r$ on $(0,1)$ for which $S(z, r)$ intersects $\partial B-E$. Choose $1>r_{1}>r_{2}>\cdots$ so that $r_{j} \rightarrow 0$ and so that

$$
l\left[J_{z} \cap\left(0, r_{j}\right)\right] \geq \frac{\delta r_{j}}{2}>2 r_{j+1}
$$

for all $j$. Then

$$
l\left[J_{z} \cap\left(r_{j+1}, r_{j}\right)\right] \geq \frac{\delta r_{j}}{4}
$$

for all $j$, which implies

$$
\int_{J_{z}} \frac{d t}{t} \geq \sum_{j=1}^{\infty} \int_{J_{z} \cap\left(r_{j+1}, r_{j}\right)} \frac{d t}{r_{j}} \geq \sum_{j=1}^{\infty} \frac{\delta}{4}=\infty,
$$

and $4^{\circ}$ follows.

To complete the proof of Theorem 7 , we verify that each of the conditions $3^{\circ}, 4^{\circ}$ and $6^{\circ}$ implies $\bmod _{B}(\partial B-E, z)$ for every $z$ in $E$ near $b$.

Assume first $3^{\circ}$. Under this assumption, in fact, $\bmod _{B}(\partial B-E, z)=$ $\infty$ for each $z$ on $\partial B$. Indeed, if $F$ is a continuum in $B$ and if $F^{*}$ denotes its image under reflection in $\partial B$, then the $O_{A D}$-condition (28) in conjunction with the minorization principle for the extremal length implies

$$
\begin{aligned}
\bmod \left[\Delta\left(F, F^{*}: \overline{\mathbb{C}}\right)\right] & =\bmod \left[\Delta\left(F, F^{*}: \overline{\mathbb{C}}-E\right)\right] \\
& \leq \bmod [\Delta(F, \partial B-E: B)]
\end{aligned}
$$


From this we infer that, given $z \in \partial B$ and $r \in(0,1)$,

$$
\liminf _{t \rightarrow 0} \bmod [\Delta(F, \partial B-E: B)]=\infty,
$$

where the infimum is taken over all continua $F$ in $B$ meeting $S(z, r)$ and $S(z, t)$. Thus $\bmod _{B}(\partial B-E, z)=\infty$ for each $z$ in $\partial B$.

Next, assume $4^{\circ}$. Standard properties of the extremal length yield

$$
\bmod [\Delta(\partial B-E, F: B)] \geq \frac{1}{\pi} \int_{J_{z} \cap(t, r)} \frac{d u}{u}
$$

for each continuum $F$ in $B$ meeting $S(z, r)$ and $S(z, t), 0<t<r \leq 1$, where $J_{z}$ is as earlier in the proof. Thus $\bmod _{B}(\partial B-E, z)=\infty$.

Finally, assume $6^{\circ}$. A technical modification in the reasoning in $\left[11\right.$, p. 771] shows that $\bmod _{\overline{\mathrm{C}}}(\partial B-E, z)=\infty$. If $F$ is a continuum in $B$, then an argument of Gehring in [7] gives

$$
\bmod [\Delta(\partial B-E, F: B)]=\bmod [\Delta(\partial B-E, F: \bar{B})]
$$

while a standard symmetry principle for the modulus yields

$$
\bmod [\Delta(\partial B-E, F: \overline{\mathbb{C}})] \leq 2 \bmod [\Delta(\partial B-E, F: \bar{B})] .
$$

Since $\bmod _{\overline{\mathrm{C}}}(\partial B-E, z)=\infty$, we infer that $\bmod _{B}(\partial B-E, z)=\infty$.

We next present an example which shows that the exceptional set $E$ in Theorem 7 can be measure-theoretically large:

THEOREM 8. There exists $a G_{\delta}$-set $E$ on $\partial B$ with

$$
\text { Hausdorff- } \operatorname{dim}(\partial B-E)=0
$$

such that

$$
\begin{aligned}
& C_{L}(f, b)=C_{L, \partial B-E}(f, b)=C_{L, \mathrm{rad}, \partial B-E}(f, b)=C_{L}\left(\dot{f} \mid\left(A_{f}-E\right), b\right), \\
& C_{R}(f, b)=C_{R, \partial B-E}(f, b)=C_{R, \mathrm{rad}, \partial B-E}(f, b)=C_{R}\left(\dot{f} \mid\left(A_{f}-E\right), b\right),
\end{aligned}
$$

and hence such that

$$
C(f, b)=C_{\partial B-E}(f, b)=C_{\mathrm{rad}, \partial B-E}(f, b)=C\left(\dot{f} \mid\left(A_{f}-E\right), b\right)
$$

for each point $b$ on $\partial B$ and for each conformal mapping $f$ of $B$.

Proof. Let $A_{0}$ be a compact Cantor-type set on $\partial B \cap\{z: \operatorname{Im} z<0\}$ of zero Hausdorff dimension and of positive capacity. (For a construction of such a set, see e.g. Wallin [14].) Next let

$$
F_{0}=\{1\} \cup\left(\bigcup_{k=0}^{\infty} h_{k}\left(A_{0}\right)\right),
$$


where $h_{k}\left(e^{i \theta}\right)=e^{i 2^{-k} \theta}$. Then $F_{0}$ is a compact set on $\partial B$ of zero Hausdorff dimension. Moreover, the lower capacity density of $F_{0}$ at 1 , cap dens $\left(F_{0}, 1\right)$, which is defined by replacing limsup by lim inf in (27), is positive. Next let $F$ designate the union of the images of $F_{0}$ under all rational rotations of $\partial B$. Then $F$ is an $F_{\sigma}$-set on $\partial B$ of zero Hausdorff dimension. It is not difficult to show that cap $\underline{\operatorname{dens}}(F, z)>0$ for each $z=e^{i \theta}$ (not only for rational $\theta$ ). The set $E=\partial B-F$ is a $G_{\delta}$-set with Hausdorff-dim $(\partial B-E)=0$ and capdens $(\partial B-E, z)>0$ for each $z$ in $\partial B$. Thus the assertions follow from Theorem 7.

8. Limits and boundary limits. A classical theorem of Lindelöf asserts that if a bounded analytic function of the unit disk $B$ is continuous up to the boundary except for one point $b$ and if the function has a limit at $b$ along the boundary, then it has the same limit at $b$ through $B[12$, p. 75]. We next record several extensions of this result in the conformal case. The first two concern an arbitrary domain $D$, while the others deal with the unit disk $B$. The results are consequences of Theorems 1, 2, 4, 6, 7 and 8 .

COROLlARY 1. Let $b$ be a non-isolated boundary point of a domain $D$ and let $f$ be a conformal mapping of $D$ admitting an extension to a continuous mapping $\bar{f}$ of $\bar{D}-\{b\}$. Then $f$ has a limit at $b$ if and only if the boundary mapping $\bar{f} \mid(\partial D-\{b\})$ has a limit at $b$.

COROLlary 2. Let $b$ be a non-isolated boundary point of a domain $D$ and let $E$ be a set on $\partial D$ such that $\bmod _{D}(\partial D-E, z)=\infty$ for each $z$ in $E \cup\{b\}$ near $b$. Next let $f$ be a conformal mapping of $D$ admitting an extension to a continuous mapping $\bar{f}$ of $\bar{D}-E$. Then $f$ has a limit $a t b$ if and only if the boundary mapping $\bar{f} \mid(\partial D-E)$ has a limit at $b$.

Corollary 3. Let $b$ be a boundary point of the unit disk $B$, let $E$ be a set on $\partial B$ and let $f$ be a conformal mapping of $B$ admitting an extension to a continuous mapping $\bar{f}$ of $\bar{B}-E$. Suppose that $\bmod _{B}(\partial B-E, z)=\infty$ for each $z$ in $E$ near $b$, or that $E$ satisfies one of the conditions $1^{\circ}-6^{\circ}$ in Theorem 6. Then $f$ has a left-hand (righthand) limit at $b$ if and only if the boundary mapping $\bar{f} \mid(\partial B-E)$ has a left-hand (right-hand) limit at $b$. Consequently, $f$ has $a$ limit at $b$ if and only if $\bar{f} \mid(\partial B-E)$ has a limit at $b$.

Corollary 4. Let $b$ be a boundary point of the unit disk $B$, let $E$ be a set on $\partial B$, let $f$ be a conformal mapping of $B$ and let $\dot{f}$ be the 
radial extension of $f$ to $B \cup A_{f}$. Suppose that $\bmod _{B}(\partial B-E, z)=\infty$ for each $z$ in $E$ near $b$, or that $E$ satisfies one of the conditions $1^{\circ}-6^{\circ}$ in Theorem 6. Then $f$ has a left-hand (right-hand) limit at $b$ if and only if $\dot{f} \mid\left(A_{f}-E\right)$ has a left-hand (right-hand) limit at $b$. Consequently, $f$ has a limit at $b$ if and only if $\dot{f} \mid\left(A_{f}-E\right)$ has a limit at $b$.

Corollary 5. There exists $a G_{\delta}$-set $E$ on $\partial B$ with

$$
\text { Hausdorff- } \operatorname{dim}(\partial B-E)=0
$$

and with the following property: Given any point $b$ on $\partial B$ and any conformal mapping $f$ of $B, f$ has a left-hand (right-hand) limit at $b$ if and only if $\dot{f} \mid\left(A_{f}-E\right)$ has a left-hand (right-hand) limit at $b$. Consequently, $f$ has a limit at $b$ if and only if $\dot{f} \mid\left(A_{f}-E\right)$ has a limit at $b$.

9. Cluster sets and Carathéodory homeomorphisms. An arbitrary homeomorphism does not satisfy any of the theorems in the present paper. In this final section, however, we exhibit a class of homeomorphisms of the unit disk $B$ for which the main theorem of this paper still holds.

It is evident that $C_{\partial B}(h, b)=C(h, b)$ for each $b$ in $\partial B$ if $h$ is a homeomorphism mapping $B$ onto a domain whose boundary degenerates to a single point. Next let $\mathscr{H}$ denote the class of homeomorphisms which map $B$ onto domains with non-degenerate boundaries. Instead of the ordinary boundary of such an image domain $D$, consider the prime end boundary $\partial^{*} D$ of $D$ obtained by adding to $D$ the prime ends of $D$ [2], [3], [13]. We say that a homeomorphism $h$ in $\mathscr{H}$ is a Carathéodory homeomorphism if it fulfills the Carathéodory Prime End Theorem; that is, $h$ can be extended to a homeomorphism between $\bar{B}$ and the prime end compactification $D \cup \partial^{*} D$ of $D=h(B)$. (Such homeomorphisms $h$ were investigated by Zorič in [15].)

THEOREM 9. Let $b$ be a boundary point of the unit disk $B$ and let $h$ be a Carathéodory homeomorphism of $B$. Then

$$
C_{L}(h, b)=C_{L, \partial B}(h, b), \quad C_{R}(h, b)=C_{R, \partial B}(h, b),
$$

and consequently,

$$
C(h, b)=C_{\partial B}(h, b) .
$$

Proof. We show that $C_{L}(h, b)=C_{L, \partial B}(h, b)$. It suffices to verify the inclusion $C_{L}(h, b) \subset C_{L, \partial B}(h, b)$. Let $\mathscr{P}$ be the prime end of $D=h(B)$ 
corresponding to $b$ under $h$. Choose an arc $\gamma$ in $B$, with an endpoint at $b$, lying to the left of the radius from $b$, such that

$$
C_{\gamma}(h, b)=C_{L}(h, b) .
$$

Here $C_{\gamma}(h, b)$ designates the cluster set of $h$ at $b$ taken along $\gamma$. The existence of such an arc $\gamma$, if not well known, can be proved as in [4, Theorem 4.6] with only trifling changes of detail, for any function $h$ (whether it is continuous or not). In the terminology of Ursell and Young [13], the path $h(\gamma)$ approaches the left wing of the impression of $\mathscr{P}$. Since $h$ satisfies the Carathéodory Prime End Theorem, (29) is valid, with $\gamma$ replaced by $\beta$, for any arc $\beta$ in $B$ which terminates at $b$ and lies between $\gamma$ and $\partial B$ (to the left of the corresponding radius). It follows, for any neighborhood $U$ of $b$, that the cluster set $C_{L}(h, b)$ is contained in the closure of $C\left(h, U_{-} \cap \partial B-\{b\}\right)$, where $U_{-}=\{z \in U: \arg z \geq \arg b\}$. This means that $C_{L}(h, b)$ is contained in $C_{L, \partial B}(h, b)$, as asserted.

\section{REFERENCES}

[1] L. V. Ahlfors and A. Beurling, Conformal invariants and function theoretic nullsets, Acta Math., 83 (1950), 125-142.

[2] C. Carathéodory, Über die Begrenzung einfachzusammenhängender Gebiete, Math. Ann., 73 (1913), 323-370.

[3] E. F. Collingwood, Cluster set theorems for arbitrary functions with applications to function theory, Ann. Acad. Sci. Fenn. A I 336/8 (1963), 1-15.

[4] E. F. Collingwood and A. J. Lohwater, The Theory of Cluster Sets, Cambridge University Press, 1966.

[5] E. F. Collingwood and G. Piranian, Asymmetric prime ends, Math. Ann., 144 (1961), 59-63.

[6] _ The mapping theorems of Carathéodory and Lindelöf, J. Math. Pures Appl., (9) 43 (1964), 187-199.

[7] F. W. Gehring, Lectures on quasiconformal mappings, Institut Mittag-Leffler, Djursholm, 1972.

[8] H. Grötzsch, Eine Bemerkung zum Koebeschen Kreisnormierungsprinzip, Berichte über die Verhandlungen der Sächsischen Akademie der Wissenschaften zu Leipzig, 87 (1936), 319-324.

[9] M. Jurchescu, Modulus of a boundary component, Pacific J. Math., 8 (1958), 791-809.

[10] O. Martio, Equicontinuity theorem with an application to variational integrals, Duke Math. J., 42 (1975), 569-581.

[11] O. Martio and J. Sarvas, Density conditions in the n-capacity, Indiana Univ. Math. J., 26 No. 4 (1977), 761-776.

[12] R. Nevanlinna, Analytic functions, Springer-Verlag, Berlin-HeidelbergNew York, 1970. 
[13] H. D. Ursell and L. C. Young, Remarks on the theory of prime ends, Mem. Amer. Math. Soc., 3 (1951), 1-29.

[14] H. Wallin, Metrical characterization of conformal capacity zero, J. Math. Anal. Appl., 58 (1977), 298-311.

[15] V. A. Zorič, Interdependence of a theorem of Koebe and a theorem of Carathédory (Russian), Mat. Zametki, 10 (1971), 399-406.

Received June 5, 1986 and in revised form December 7, 1988. Part of this research was done while the author was visiting The University of Texas at Austin in 1985-86.

The University of TeXas at Austin

Austin, TX 78712-1082

AND

UNIVERSITY OF JYVÄSKYLÄ

JYVÄSKYLÄ, FINLAND 


\section{PACIFIC JOURNAL OF MATHEMATICS EDITORS}

\author{
V. S. VARADARAJAN \\ (Managing Editor) \\ University of California \\ Los Angeles, CA 90024-1555-05 \\ Herbert Clemens \\ University of Utah \\ Salt Lake City, UT 84112 \\ THOMAs ENRIGHT \\ University of California, San Diego \\ La Jolla, CA 92093
}

R. FINN

Stanford University

Stanford, CA 94305

HermanN FlaschKa

University of Arizona

Tucson, AZ 85721

VAughan F. R. Jones

University of California

Berkeley, CA 94720

STEVEN KeRCKHOFF

Stanford University

Stanford, CA 94305

\author{
RobION KIRBY \\ University of California \\ Berkeley, CA 94720 \\ C. C. MoOre \\ University of California \\ Berkeley, CA 94720
}

Harold Stark

University of California, San Diego

La Jolla, CA 92093

\section{ASSOCIATE EDITORS
R. ARENS
E. F. BECKENBACH
B. H. NeumanN
F. WoLF
K. YoshidA (1906-1982)}

\section{SUPPORTING INSTITUTIONS}

UNIVERSITY OF ARIZONA
UNIVERSITY OF BRITISH COLUMBIA
CALIFORNIA INSTITUTE OF TECHNOLOGY
UNIVERSITY OF CALIFORNIA
MONTANA STATE UNIVERSITY
UNIVERSITY OF NEVADA, RENO
NEW MEXICO STATE UNIVERSITY
OREGON STATE UNIVERSITY

\author{
UNIVERSITY OF OREGON \\ UNIVERSITY OF SOUTHERN CALIFORNIA \\ STANFORD UNIVERSITY \\ UNIVERSITY OF HAWAII \\ UNIVERSITY OF TOKYO \\ UNIVERSITY OF UTAH \\ WASHINGTON STATE UNIVERSITY \\ UNIVERSITY OF WASHINGTON
}

The Supporting Institutions listed above contribute to the cost of publication of this Journal, but they are not owners or publishers and have no responsibility for its content or policies.

Mathematical papers intended for publication in the Pacific Journal of Mathematics should be in typed form or offset-reproduced (not dittoed), double spaced with large margins. Please do not use built up fractions in the text of the manuscript. However, you may use them in the displayed equations. Underline Greek letters in red, German in green, and script in blue. The first paragraph must be capable of being used separately as a synopsis of the entire paper. In particular it should contain no bibliographic references. Please propose a heading for the odd numbered pages of less than 35 characters. Manuscripts, in triplicate, may be sent to any one of the editors. Please classify according to the scheme of Math. Reviews, Index to Vol. 39. Supply name and address of author to whom proofs should be sent. All other communications should be addressed to the managing editor, or Elaine Barth, University of California, Los Angeles, California 90024-1555-05.

There are page-charges associated with articles appearing in the Pacific Journal of Mathematics. These charges are expected to be paid by the author's University, Government Agency or Company. If the author or authors do not have access to such Institutional support these charges are waived. Single authors will receive 50 free reprints; joint authors will receive a total of 100 free reprints. Additional copies may be obtained at cost in multiples of 50 .

The Pacific Journal of Mathematics is issued monthly as of January 1966. Regular subscription rate: $\$ 190.00$ a year (5 Vols., 10 issues). Special rate: $\$ 95.00$ a year to individual members of supporting institutions.

Subscriptions, orders for numbers issued in the last three calendar years, and changes of address should be sent to Pacific Journal of Mathematics, P.O. Box 969, Carmel Valley, CA 93924, U.S.A. Old back numbers obtainable from Kraus Periodicals Co., Route 100, Millwood, NY 10546.

The Pacific Journal of Mathematics at P.O. Box 969, Carmel Valley, CA 93924 (ISSN 0030-8730) publishes 5 volumes per year. Application to mail at Second-class postage rates is pending at Carmel Valley, California, and additional mailing offices. Postmaster: send address changes to Pacific Journal of Mathematics, P.O. Box 969, Carmel Valley, CA 93924.

\section{PUBLISHED BY PACIFIC JOURNAL OF MATHEMATICS, A NON-PROFIT CORPORATION}

Copyright (C) 1990 by Pacific Journal of Mathematics 


\section{Pacific Journal of Mathematics}

\section{Vol. 141, No. 2 December, 1990}

Ulrich F. Albrecht, Locally $A$-projective abelian groups and

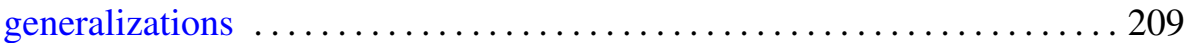

Michel Carpentier, Sommes exponentielles dont la géométrie est très belle: $p$-adic estimates ..................................... 229

G. Deferrari, Angel Rafael Larotonda and Ignacio Zalduendo, Sheaves and functional calculus

Jane M. Hawkins, Properties of ergodic flows associated to product odometers ........................................287

Anthony To-Ming Lau and Viktor Losert, Complementation of certain subspaces of $L_{\infty}(G)$ of a locally compact group ............... 295

Shahn Majid, Matched pairs of Lie groups associated to solutions of the

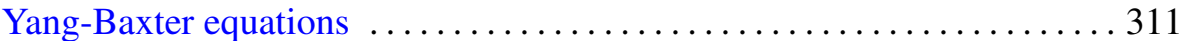

Diego Mejia and C. David (Carl) Minda, Hyperbolic geometry in $k$-convex

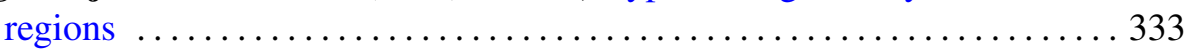

Vladimír Müller, Kaplansky's theorem and Banach PI-algebras ...........355

Raimo Näkki, Conformal cluster sets and boundary cluster sets coincide . . . 363

Tomasz Przebinda, The wave front set and the asymptotic support for $p$-adic groups

R. F. Thomas, Some fundamental properties of continuous functions and topological entropy 